\title{
Percepción del servicio de agua de los acueductos comunitarios de la comuna 13 de Ibagué
}

\author{
Perception of water service for community water supply of the \\ commune 13 Ibague.
}

\author{
Alexander Pinilla Carvajal ${ }^{1}$, Hildebrando Ramírez Arcila ${ }^{2}$, \\ Laura Constanza Gallego Cossio ${ }^{3}$ \\ ${ }^{I} M S c$ (c). Facultad de Ingeniería, Universidad Cooperativa de Colombia, Ibagué, Colombia \\ alexander.pinilla@campusucc.edu.co \\ ${ }^{2}$ M Sc. Facultad de Ingeniería, Universidad Cooperativa de Colombia, Ibagué, Colombia \\ hildebrando.ramirez@ campusucc.edu.co \\ ${ }^{3}$ M BA. Facultad de Contaduría Pública, Universidad Cooperativa de Colombia, Ibagué, Colombia \\ laura.gallego@campusucc.edu.co
}

\begin{abstract}
Resumen- Los acueductos comunitarios se han encargado de gestionar el uso del recurso hídrico con fines de consumo humano y doméstico. Estas organizaciones, alcanzan a suplir esta necesidad básica aproximadamente en un $20 \%$ de la población nacional. Esta gran responsabilidad que no puede estar por fuera del control gubernamental, tiene implicaciones sustanciales en la salud pública $y$ en el ambiente que las hacen motivo de preocupación por parte de los investigadores. La presente investigación tuvo como objetivo identificar las percepciones que tienen los habitantes de la comuna 13 de la ciudad de Ibagué sobre el servicio de agua potable que prestan los acueductos comunitarios que los abastecen. Este estudio descriptivo se realizó mediante el método de estudio de caso y pudo establecer que los usuarios de este servicio son conscientes de las deficiencias que presenta $y$ de las posibles implicaciones negativas que puede tener para la salud de las personas.
\end{abstract}

Palabras clave- Acueductos comunitarios, calidad del agua, salud pública, percepciones.

Abstract - Community water systems have been commissioned to manage the use of water intended for human consumption and domestic. These organizations manage to meet this basic need approximately $20 \%$ of the national population. This great responsibility that cannot be outside of government control, has substantial implications for public health and the environment that make concern by researchers. This research aimed to identify the perceptions of the inhabitants of the municipality 13 Ibagué on the drinking water provided by community water systems that supply them. This descriptive study was conducted using the case study method and was able to provide that service users are aware of the deficiencies and possible negative implications it may have on the health of people.

Fecha de Recepción: 14 de febrero de 2016

Fecha de Aceptación: 23 de Noviembre de 216
Key Word - Community water systems, water quality, public health, perceptions.

\section{INTRODUCCIÓN}

El municipio de Ibagué está ubicado en el centro del departamento del Tolima, cercano a la cordillera central. Posee una población aproximada a los 560.000 habitantes. Tiene una superficie de $1439 \mathrm{~km} 2$, es la capital del departamento con el mayor número de población y lugar donde se concentra la mayor parte de las actividades industriales del mismo.

Este municipio presenta una grave problemática relacionada con el manejo y aprovechamiento de los recursos hidráulicos, en particular lo relacionado con el agua potable para la población más vulnerable. En este grupo se encuentra la población ubicada en la comuna 13 la cual se encuentra ubicada partiendo de la carretera Ibagué - Armenia por la tejada el Tejar aguas abajo, hasta la desembocadura de la quebrada la Volcana cerca al cementerio militar, hasta un sitio conocido como El Boqueron donde parte la carretera hacia los corregimientos de Toche y Tapias, a una cota de altura comprendida entre 1250 - $1400 \mathrm{msnm}$, el abastecido de agua potable es un limitante para el desarrollo, en su mayoría existen casas de un solo piso y un significativo número de áreas en riesgo de deslizamiento.

La necesidad de suplir las necesidades de saneamiento básico ha favorecido la creación de acueductos comunitarios que en su mayoría toman el agua de las quebradas circundantes y 
retornan los residuos al mismo con un escaso tratamiento, lo cual se convierte en un inconveniente para la sanidad del sector y sus habitantes. Otro problema detectado es el uso v creciente y desordenado de los asentamientos que en su mayoría se encuentran en la cercanía de los causes de las quebradas favoreciendo la contaminación y poniendo en riesgo la seguridad de quienes allí viven.

Los problemas de abastecimiento de agua, en gran parte del municipio de Ibagué, han sido resueltos gracias a la incorporación de las aguas del Acueducto Regional del Centro a la red de abastecimiento municipal, sin embargo, los sectores ubicados en las zonas de mayor altitud no se benefician por este servicio y los problemas ambientales mencionados cada vez son peores.

Los acueductos comunitarios son entidades populares desarrolladas en torno a la gestión del agua para consumo humano en pequeñas colectividades como son veredas, resguardos indígenas, comunidades negras y algunos barrios de diferentes ciudades del país como se menciona en [1]. Estas entidades presentan un alto grado de complejidad toda vez que su construcción obedece a un contexto socioeconómico particular y variado que corresponde a la complejidad misma del trabajo comunitario [2].

Es importante tener en cuenta que el agua es un recurso natural que hace parte del patrimonio nacional y por lo tanto es un bien de propiedad común, es "un recurso natural limitado y un bien público fundamental para la vida y la salud" [3]. No obstante, al cierre del quinto foro mundial del agua no fue posible unificar la posición sobre su propiedad, solamente fue posible establecer que el agua es una "necesidad básica" [4]. En este sentido, los acueductos comunitarios asumen la gestión de este recurso y por ende la salud pública [5], por cuanto este elemento contaminado puede contribuir a la presentación de diversas enfermedades de diverso tipo en los consumidores [6], especialmente las de origen parasitario o infeccioso [7,8]. Esta responsabilidad, es reconocida en estos acueductos dependiendo de la categoría que se les otorga en la escala nacional, regional o local, del manejo ambiental que le compete, de su carácter y alcances asociativos, de su dimensión pública o privada, y de las formas concretas de su legitimidad. En algunos casos, estas empresas son de carácter familiar, vecinal, empresarial, artesanal y algunas presentan diversas formas de organización. El reconocimiento de hecho y/o de derecho de estas entidades por parte del gobierno se presenta de manera desigual [9]; sin embargo, estos acueductos son forzados unificadamente a adelantar procesos de formalización en cumplimiento de la ley vigente de servicios públicos [10].

Los sistemas comunitarios de abastecimiento de agua, en la mayoría de los casos, no son eficientes y deben enfrentar diversas problemáticas que han sido objeto de estudio de diversas investigaciones [11]. Si bien es cierto que los acueductos prestan un servicio público, no siempre estas empresas son del estado; sin embargo, es responsabilidad del gobierno asegurar el beneficio general [12].

Se han podido identificar, por ejemplo, el desconocimiento de la capacidad y de la calidad de las fuentes superficiales y subterráneas de agua. La falta de conciencia en lo referido a la contaminación ambiental derivada del vertido directo de las aguas servidas al suelo, ríos y quebradas. La limitada infraestructura -bocatomas, tanques de almacenamiento y redes- frente a las grandes demandas de agua potable. La inexistencia y resistencia al uso de medidores domiciliarios. La cultura de la morosidad acompañada de sistemas tarifarios obsoletos. Los desperdicios en el sistema general y en las conexiones domiciliarias. La falta de sensibilización, concienciación ciudadana, educación ambiental y desconocimiento del marco legal, así como la débil formación de organizaciones comunitarias. El objeto del presente trabajo fue identificar la percepción que tienen los habitantes de la comuna 13 de la ciudad de Ibagué sobre el servicio de agua potable que les suministran los acueductos comunitarios.

\section{CONTENIDO}

Los sistemas comunitarios de abastecimiento de agua, en la mayoría de los casos, no son eficientes y deben enfrentar diversas problemáticas que han sido objeto de estudio de diversas investigaciones [13]. Si bien es cierto que los acueductos prestan un servicio público, no siempre estas empresas son del estado; sin embargo, es responsabilidad del gobierno asegurar el beneficio general [12].

Se han podido identificar, por ejemplo, el desconocimiento de la capacidad y de la calidad de las fuentes superficiales y subterráneas de agua. La falta de conciencia en lo referido a la contaminación ambiental derivada del vertido directo de las aguas servidas al suelo, ríos y quebradas. La limitada infraestructura -bocatomas, tanques de almacenamiento y redes- frente a las grandes demandas de agua potable. La inexistencia y resistencia al uso de medidores domiciliarios. La cultura de la morosidad acompañada de sistemas tarifarios obsoletos. Los desperdicios en el sistema general y en las conexiones domiciliarias. La falta de sensibilización, concienciación ciudadana, educación ambiental y desconocimiento del marco legal, así como la débil formación de organizaciones comunitarias.

Todas estas situaciones, pueden llevar a comprometer la prestación de un servicio público que, en lo correspondiente a los acueductos comunitarios, aproximadamente suple de agua potable al $20 \%$ de los habitantes de las ciudades en Colombia; sin embargo, esta proporción no siempre se conserva en las diferentes regiones [14]. Como sucede en otros municipios, Ibagué tiene acueductos comunitarios en los cuales se presume que se presentan las diversas problemáticas que han sido identificadas en otras regiones del país. En esta ciudad, existen 28 acueductos comunitarios que surten de agua a diversos barrios periféricos de la ciudad, a partir del agua 
proveniente de 22 micro-cuencas (Tabla 1). Estos acueductos comunitarios, tienen una cobertura del $20 \%$ del servicio total de la ciudad. En el sector rural, la cobertura del servicio prestado por estos acueductos alcanza el 40\%. Sin embargo, no hay suficiente información que permita establecer claramente cuales son específicamente éstas dificultades y en consecuencia, es difícil llegar a proponer estrategias que contribuyan, en el caso de ser necesario, al mejoramiento de la prestación de este servicio público en los sectores en donde se encuentran inmersos.

\section{METODOLOGÍA}

El presente estudio fue abordado desde el enfoque cualitativo, por cuanto el interés fue describir un fenómeno social desde el sentir de sus protagonistas. El método utilizado fue un estudio de caso descriptivo transeccional.

El estudio de caso es apropiado para abordar enfoques cualitativos [15]. Este método, permite estudiar un fenómeno en su contexto espacio temporal, aunque las fronteras entre el fenómeno y el entorno no estén claramente definidas [16]. El estudio de caso puede ser "una cultura, una sociedad, una comunidad, una subcultura, una organización, un grupo o fenómenos tales como creencias, prácticas o interacciones, así como cualquier aspecto de la existencia humana" [17]. El análisis descriptivo permite identificar la percepción que tienen los habitantes de la comuna 13 de la ciudad de Ibagué con respecto a la calidad del servicio de agua que reciben de sus respectivos acueductos comunitarios.

Para obtener los datos necesarios se realizó una encuesta. Esta técnica, permite obtener información sobre el objeto de estudio y es de "gran utilización en investigaciones teóricas y aplicadas a la sociología, la psicología social, la ciencia política, la demografía, la educación [18].

La muestra se determinó a partir de una población de 16035 habitantes. Dicha muestra, fue obtenida mediante la aplicación de la siguiente fórmula, con un índice de confianza del $95 \%$ y una probabilidad positiva y negativa equivalente al $50 \%$ para $\operatorname{ambos} \operatorname{casos}(\mathrm{p}=\mathrm{q}=0.5)$.

$n=\frac{Z^{2} * p * \mathrm{q} * \mathrm{~N}}{N * E^{2}+Z^{2} * p * q}$

Donde $\mathrm{n}$ es el tamaño de la muestra; $\mathrm{Z}$ es el nivel de confianza; $p$ es la variabilidad positiva; $q$ es la variabilidad negativa; $\mathrm{N}$ es el tamaño de la población; E es la precisión o el error.

$n=375 n=\frac{(1.96)^{2} *(0.5) *(0.5) *(16035)}{(16035) *(0.05)^{2}+(1.96)^{2} *(0.5) *(0.5)}$
De esta forma la muestra fue determinada en 375 personas.

\section{RESULTADOS Y DISCUSIÓN}

Mediante la aplicación del instrumento de recolección se pudo determinar que el $70 \%$ de la muestra corresponde al género femenino y el $30 \%$ corresponde al género masculino. Las personas que habitan las viviendas se distribuyen en adultos en un $59 \%$ y menores de edad $41 \%$.

En cuanto al suministro de agua potable se pudo determinar que el $62 \%$ de los habitantes encuestados tiene la percepción de que el agua que reciben es de calidad mala o regular; no obstante, el restante $38 \%$ considera que la calidad del agua es buena o excelente. El 59\% de los usuarios confía en la organización comunitaria para enfrentar cualquier situación que pudiera llegar a afectar negativamente su acueducto. También, pudo establecerse que el $65 \%$ de los usuarios estarían dispuestos a pagar un mayor valor por el servicio de agua, con tal de recibir un producto de mejor calidad. En esta misma categoría, el $78 \%$ de los encuestados cree que al mejorar la calidad del agua que se utiliza para el consumo humano, se disminuye el riesgo de contraer enfermedades.

En cuanto al proveedor del servicio de agua, se pudo determinar que el $57 \%$ de los usuarios prefieren escogerlo por la calidad que ofrecen. No obstante, el $26 \%$ lo escoge por el costo, el $9 \%$ por ser comunitario, el $3 \%$ por la continuidad del servicio y el $5 \%$ no tienen un criterio de selección definido. Sin embargo, el $71 \%$ de los encuestados considera que la elección del proveedor depende más de la situación económica del usuario.

En cuanto a la posibilidad de la construcción de un acueducto alterno de carácter gubernamental se pudo determinar que el $75 \%$ de los encuestados considera que esta obra sería muy importante para la comunidad por cuanto se recibiría un producto seguro para consumir. No obstante, esta misma proporción manifiesta su preocupación por el costo que puede llegar a tener este servicio en otro acueducto. Igualmente les preocupa que al ser implementado otro acueducto se abandonen las instalaciones del comunitario, el cual ha sido construido con tanto esfuerzo.

El $63 \%$ de los encuestados cree que sería útil, para el servicio de agua potable, la instalación de micro-medidores que en consecuencia se realizaría con la implementación de esta nueva obra. También, el $68 \%$ de los encuestados cree que con la instalación de micro-medidores sería posible racionalizar el servicio de agua 


\begin{tabular}{|c|c|c|c|c|}
\hline Área urbana & Comuna & $\begin{array}{l}\text { Acueductos comunitarios- } \\
\text { barrios }\end{array}$ & Total & $\begin{array}{l}\text { Fuente (quebrada o } \\
\text { micro-cuenca) }\end{array}$ \\
\hline \multirow[t]{10}{*}{ Sur } & \multirow[t]{10}{*}{13} & Boquerón, Ricaurte & \multirow[t]{10}{*}{15} & Q. El Tejar \\
\hline & & Florida, Batallón & & Q. La Volcana \\
\hline & & Los Túneles, La Isla, La Unión & & Q. La Tigrera \\
\hline & & Jazmín Baja & & Q. El Salero \\
\hline & & Jazmín Alta & & Q. El Salerito \\
\hline & & San Isidro, Granada & & Q. Granate \\
\hline & & Colinas I & & Q.La esmeralda \\
\hline & & Colinas II & & Q. La Cristalina \\
\hline & & Darío Echandía & & Pozos D. E. \\
\hline & & Miramar & & Q. La Gallinaza \\
\hline \multirow[t]{6}{*}{ Norte } & \multirow[t]{6}{*}{6} & Gaviota & \multirow[t]{6}{*}{7} & La Tuza \\
\hline & & El Triunfo, Los Ciruelos & & Q. Ambalá \\
\hline & & Ambalá & & Las Panelas \\
\hline & & Las Delicias & & La Balsa \\
\hline & & San Antonio & & Q.San Antonio \\
\hline & & Modelia & & Q. Cocare \\
\hline \multirow[t]{4}{*}{ Nor-occidental } & \multirow[t]{3}{*}{2} & $\mathrm{La} \mathrm{Paz}$ & \multirow[t]{3}{*}{3} & Q. La Aurora \\
\hline & & Clarita Botero & & Q. Madroño \\
\hline & & Santa Cruz & & Q. Pañuelo \\
\hline & 3 & Calambeo & 1 & Q. Grande \\
\hline \multirow[t]{2}{*}{ Centro } & \multirow[t]{2}{*}{1} & La Vega & \multirow[t]{2}{*}{2} & Q. Lavapatas \\
\hline & & Chapetón & & Q. Ramos Asti... \\
\hline Totales & & & 28 & 22 \\
\hline
\end{tabular}

Fuente: Alcaldía municipal de la ciudad de Ibagué-Empresa Ibaguereña de Acueducto y Alcantarillado S.A. ESP Oficial.

Tabla 1. Acueductos comunitarios de la ciudad de Ibagué

\section{CONCLUSIONES}

La percepción de los habitantes de la comuna 13 de la ciudad de Ibagué sobre el servicio de abastecimiento de agua potable, en su mayoría, es que el agua que consumen no es potable y reconocen que el acueducto comunitario presenta deficiencias.

De la misma forma, una proporción mayoritaria preferiría pagar un costo un poco mayor por este servicio con tal de recibir un producto de mejor calidad.

Todas las captaciones se encuentran dentro de la región perimetral de la comuna 13 , por lo cual las actividades dentro del mismo deben regirse por el Plan de Ordenamiento territorial y Uso respectivo.

A pesar de la importancia de la zona por sus recursos hídricos no existen un seguimiento adecuado en la zona actualmente operativas. consumir agua que no reúna las condiciones de calidad, aumenta el riesgo de contraer enfermedades gástricas, intestinales e infecciosas. Igualmente reconocen, que la implementación de un acueducto gubernamental y la instalación de micro-medidores permitirían mejorar la calidad del servicio y racionalizar el uso de agua, evitando desperdicios y consumos exagerados. Las conclusiones son obligatorias y deben ser claras. Deben expresar el balance final de la investigación o la aplicación del conocimiento.

\section{AGRADECIMIENTOS}

Los autores expresan su agradezco a la Dirección de Investigaciones de la Universidad Cooperativa de Colombia, por el apoyo suministrado para el desarrollo de esta investigación.

\section{REFERENCIAS}


[1]. A. Quintana, "La gestión del acueducto en Dosquebradas Risaralda, una historia de autogestión y privatización". Luna Azul, vol 30, 164-173, 2010.

[2]. Rodríguez, R., Bellido, O., González, C., Solares, E., Rojas, I., \& Lorenzo, R. "Mejoramiento sostenible de la calidad de vida de la población mediante el trabajo comunitario". Revista cubana de salud pública, vol 37 (3), 219-229, 2011.

[3]. H. Hernández, \& J. Méndez, "Viabilidad económica del mínimo vital de agua potable en la ciudad de Bogotá D. C". Producción +Limpia, vol 8(1), 102118, 2013.

[4]. P. García, \& N. Obregón, Quindío, "Consideraciones de ética ambiental en la gestión integral del recurso hídrico de la cuenca del río". Entramado, vol 8(2), 12-37, 2012.

[5]. Y. Barrientos, C. Suárez, S. Ruiz, B. Devia, \& Y. Perdomo, "Calidad Microbiológica Del Agua Y Riesgo Sanitario De Dos Acueductos Rurales En El Estado Vargas", Venezuela. Investigación y postgrado, vol 20(1), 2005.

[6]. J. Hernández, "Potabilidad del agua en Acueductos de San Juan y Martínez Provincia Pinar del Río. 2009- 2010". Revista de Ciencias Médicas de Pinar del Río, vol 16(3), 2012.

[7]. J. Londoño, A. Polo, \& C. Vergara, "Parasitismo intestinal en hogares comunitarios de dos municipios del departamento del Atlántico, norte de Colombia". Boletín de malariología y salud ambiental, vol 1(2), 251-260, 2010.

[8]. D. Mora, C. Portuguez, "Diagnóstico de la cobertura y calidad del agua para consumo humano en Costa Rica a principios del año 2000". Revista costarricense de salud pública, vol 9(16), 2000.

[9]. I. Gómez, "La acción colectiva del agua en Colombia y el referendo como acercamiento de democracia directa". Análisis político, vol 27(80), 79-103, 2014.

[10]. J. Moncada, C. Pérez, \& G. Valencia, "Comunidades organizadas y el servicio público de agua potable en Colombia: una defensa de la tercera opción económica desde la teoría derecursos de uso común". Ecos de economía, pp. 125-159, 2013.

[11]. C. Valiente, "Vigilancia sanitaria del agua: un nuevo enfoque para municipalidades de Costa
Rica". Revista Costarricense de Salud Pública, 8(15). (diciembre de 1999).

[12]. J. Amar, \& J. Echeverría, "Participación comunitaria para el control ciudadano de los servicios públicos domiciliarios". Revista de derecho, vol 29, 129-152, 2008.

[13]. Valiente, C. "Vigilancia sanitaria del agua: un nuevo enfoque para municipalidades de Costa Rica". Revista Costarricense de Salud Pública, vol 8 (15), 2009.

[14]. N. Rodríguez, S. Restrepo, \& I. Zambrano, "Carencia de agua y sus implicaciones en las prácticas alimentarias, en Turbo, Antioquia". Revista de salud pública, vol 15(3), 446-454, 2013.

[15]. Hernández, R., Fernández, C., \& Baptista, P. Metodología de la investigación. Méjico: MCGRAW-HILL. (1997).

[16]. P. Martinez, "El método de estudio de caso. Estrategia metodológica de la investigación científica". Pensamiento y gestión, 165-193, 2006.

[17]. C. Sandoval, "Investigación cualitativa". Bogotá: ARFO Editores e Impresores Ltda, 2002.

[18]. G. Briones, "Metodología de la investigación cuantitativa en las ciencias sociales". Bogotá: ARFO Editores e Impresores Ltda, 2002. 